\title{
La cirugía del carcinoma de esófago torácico
}

\author{
J. Fernández-Llamazares, F. García Cuyas, A. Font
}

\section{Introducción}

La mayor parte de los carcinomas esofágicos son de tipo escamoso. Su frecuencia resulta muy variable, de unos 20 por 100.000 habitantes en Estados Unidos y Europa a 160 por 100.000 habitantes en ciertas ciudades de China y de Sudáfrica'. Los factores ambientales que influyen en estas altas frecuencias aún no han sido identificados de forma concluyente.

El adenocarcinoma de esófago, antes de rara frecuencia, está creciendo a ritmo alarmante en el mundo occidental, principalmente por aumento en la parte distal del esófago, representando en algunas series más del 50\% del cáncer esofágico.

El cáncer de esófago suele descubrirse cuando se presentan síntomas y ello implica el diagnóstico en una etapa avanzada de la enfermedad. La frecuencia de invasión de ganglios linfáticos es alta, la diseminación submucosa del tumor y la extensión a estructuras circundantes condiciona el tratamiento cuando se diagnostica (Akiyama Ann Surg. 1994).

La cirugía radical sin tumor residual (RO) más linfadenectomía loco regional, es el tratamiento de elección de los pacientes con cáncer de esófago localizado. Sin embargo, a pesar de la resección completa del tumor y la linfadenectomía extensa ${ }^{2,3}$, son comunes las recurrencias locales y sistémicas, con unas tasas de supervivencia a los cinco años (de los pacientes resecados), que varían del 10 al 39\%. La supervivencia en los pacientes tratados únicamente con cirugía está entre el 8 y $20 \%$, dependiendo del estadiaje. La supervivencia depende en gran manera del estadio ${ }^{4}$ en el que se realiza el diagnóstico. En los países occidentales, el 70\% de los pacientes diagnosticados de cáncer esofágico se realiza en estadio III según la clasificación de TNM (T3 o T4 con N+) de la World Health Organization (WHO), a los 5 años la supervivencia post resección es menor del 15\%. Actualmente, la valoración de los factores de riesgo ${ }^{5,6}$, la indicación qui-

Hospital General Universitario Germans Trias i Pujol Badalona (Barcelona) rúrgica más selectiva y los cuidados postoperatorios han hecho que la mortalidad post esofagectomía disminuya cerca del $5 \%$ en centros de referencia ${ }^{7}$.

El tratamiento clásico del cáncer esofágico es la esofagectomía, pero debido a la alta incidencia de recidiva local o a distancia, es necesario asociar al tratamiento quirúrgico otros tratamientos adyuvantes ${ }^{8}$. La quimioterapia administrada de forma preoperatoria puede incrementar la resecabilidad mediante la disminución del tamaño tumoral y a la vez evitar la aparición de micro metástasis subclínicas. En la mayoría de estudios se obtienen respuestas ente el 40 y $66 \%$ utilizando quimioterapia preoperatoria (Cisplatino/Fluoracilo) (5-Fu). De todos modos, la respuesta patológica completa (RPC) no sobrepasa el 10\%. Se han estudiado diferentes estrategias para potenciar el efecto de a quimioterapia frente al cáncer esofágico; de esta manera, en estudios in vitro, se ha visto que la asociación de ácido fólico al 5-Fu hace que aumente su citotoxicidad, como se ha podido demostrar en tumores de cabeza y cuello.

En la actualidad, la quimioterapia y la radioterapia preoperatorias $^{9-16}$ ofrecen las mejores expectativas. En el cáncer esofágico la asociación de quimioterapia y radioterapia ha demostrado un mayor beneficio frente a la radioterapia sola (27\% de supervivencia a los 5 años frente al $0 \%$ respectivamente). Con la quimiorradioterapia preoperatoria se llegan a conseguir RPC entre el 20 y $40 \%$. La mayor parte de los estudio basados en quimioterapia y radioterapia preoperatoria utilizan la combinación de cisplatino/5. Fu y entre 40 $45 G y$ de radiación, aunque en la actualidad la asociación preoperatoria más adecuada aún no está establecida.

Nuestra experiencia en el Hospital Universitario "Germans Trias i Pujol" de Badalona, Barcelona, data de 1984. Hasta 2003 un mismo Grupo Cáncer de Esófago H.U.G.TiP. ${ }^{17}$, integrado por endoscopista, radiólogo, oncólogo, radioterapeuta, dietista, intensivista, anestesista y cirujanos (2); con criterios que han ido evolucionando en el tiempo han tratado un total de 408 pacientes afectos de neoplasia de esófago habiendo sido resecados 198 tumores, (49\%) (Fig. 1). En el momento del diagnóstico, el Estadio $\mathrm{I}-\|=5 \%$, Estadio III=59\%, Estadio IV=36\%.

En los pacientes, la edad media de 65 años con un rango (30-95), siendo el $92 \%$ hombres y el $8 \%$ mujeres. Los tumores escamosos 338 casos (83\%) y los adenocarcinomas 70 casos (17\%); llama la atención en los últimos años el incremento al 33\% en nuestros casos (Fig. 2). 


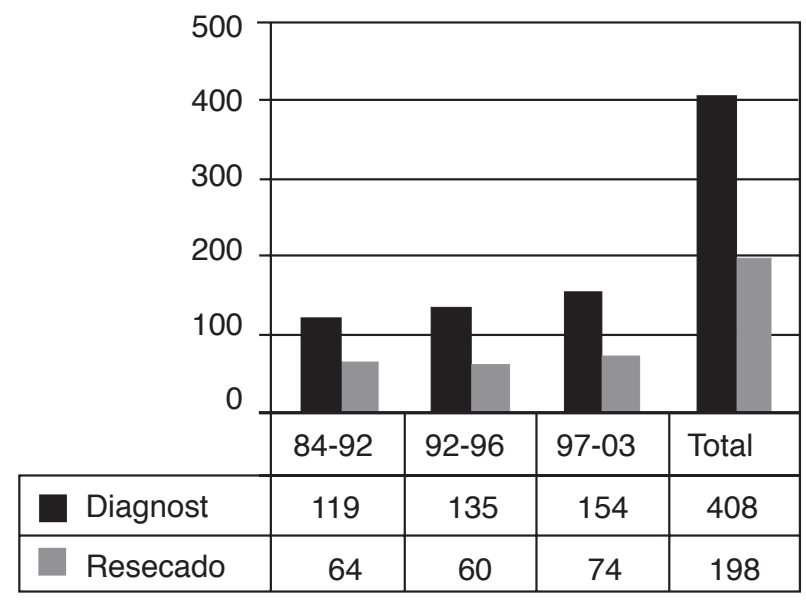

Fig. 1.

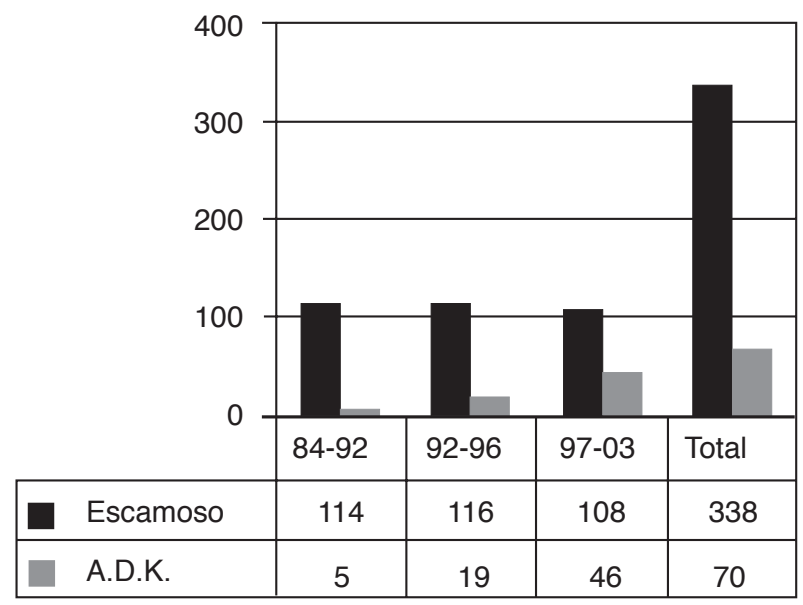

Fig. 2.

Nuestra serie se divide en tres etapas, debido a que en el tiempo la indicación terapéutica se ha ido modificando.

En la primera etapa, del 84 al 91, el tratamiento fue únicamente quirúrgico, con un total de 119 casos, de los cuales, 64 fueron resecados (54\%), con una mortalidad del 13,5\% y una supervivencia a los cinco años del $14 \%$.

En la segunda etapa, 92-96, con intención de mejorar la tasa de supervivencia y de resección, iniciamos el Protocolo terapéutico prospectivo con Quimioterapia preoperatoria ${ }^{18,19}$ (Cisplatino+5 Fluoracilo) en los tumores escamosos y después cirugía; 116 casos de los cuales 58 fueron resecados $(50 \%)$ con una mortalidad del $11 \%$ y una supervivencia a los cinco años de13\%. Dichos resultados no mejoraron nuestra experiencia previa a pesar de haber obtenido una tasa de respuesta parcial en el $30 \%$ de los casos. Esta no se correlacionó con una mayor supervivencia de los pacientes.

A pesar de que muchos autores mezclan en sus series tumores escamosos y adenocarcinomas, nuestro grupo establece diferencias entre los dos grupos. Desde 1984 a 2003 han sido tratados 70 pacientes con adenocarcinoma esofágico, destacando su incremento en la última década. Estos han si-
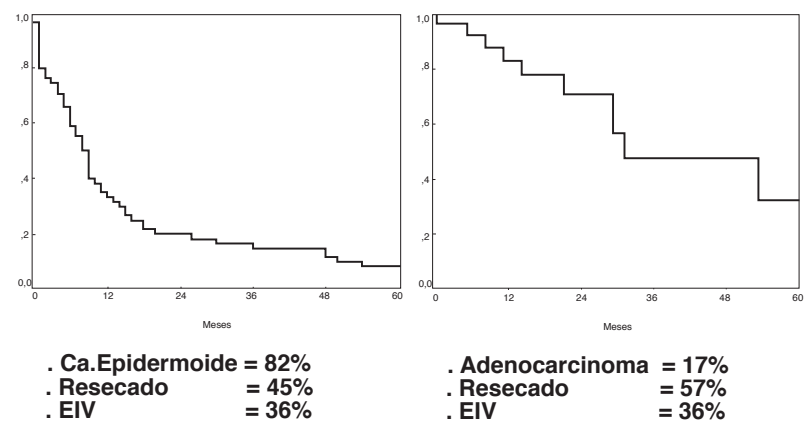

Fig. 3.

do tratados sólo quirúrgicamente, resecando 40 (57\%) con una mortalidad $7,5 \%$ y una supervivencia a los cinco años del $27 \%$. Supervivencia ${ }^{20}$ comparada (Fig. 3).

Diferentes estudios ponen en evidencia avances en la terapia coadyuvante en estos pacientes; la radioterapia hiperfraccionada mejora el control local del tumor esofágico frente a la radioterapia estándar (Nishimura 1994). La infusión continua de 5-Fluoracilo con radioterapia concurrente demuestra gran actividad frente al carcinoma escamoso esofágico (Sakai 1995). En el cáncer esofágico, la quimioterapia preoperatoria junto a la radioterapia aumenta la supervivencia en comparación con la cirugía sin tratamiento adyuvante ${ }^{15}$.

\section{Objetivo}

Una tercera etapa desde 1997 hasta 2003, a raíz de los resultados expuestos por diferentes grupos y con el objetivo de mejorar la supervivencia, nuestro Grupo Cáncer de Esófago H.U.G.TiP. ha diseñado una combinación preoperatoria de cisplatino semanal e infusión continua de fluoracilo junto a radioterapia hiperfraccionda en el cáncer escamoso esofágico.

\section{Protocolo terapéutico con quimio+radioterapia hiperfraccionada preoperatoria+cirugía}

El principal objetivo de este estudio es la valoración de este esquema terapéutico basado en la resecabilidad del tumor, respuesta anatomopatológica, tiempo de progresión y supervivencia.

En segundo lugar, definir la toxicidad de este tratamiento y el impacto en el riesgo postoperatorio.

\section{Método}

El paciente con sospecha de cáncer de esófago ingresa en el servicio de Cirugía, se practica una endoscopia digestiva alta con biopsia; ante una biopsia positiva para carcinoma se procede al estudio general del paciente y estudio de extensión de la lesión, según Tabla I. La enfermedad metastásica (36\% de nuestros casos) pasa a valoración por Oncología Médica.

En los pacientes que la enfermedad se cataloga de locoregional esofágica se realiza ecoendoscopia, pruebas funcionales respiratorias y fibrobroncoscopia en tumores localizados por encima de la carina bronquial. 
TABLA I

\begin{tabular}{ll}
\hline & Evaluación pre tratamiento \\
\hline Clínica & De la lesión \\
\hline Examen Clínico & Fibrogastroscopia+biopsia \\
Rx Tórax & TC toraco abdominal \\
E.C.G. & Ecografía hepática \\
Analítica general & Broncoscopia \\
Bioquímica & Ecoendoscopia esofágica \\
Pruebas funcionales respiratorias & Tránsito esofágico \\
\hline
\end{tabular}

Los pacientes afectos de un adenocarcinoma esofágico en estadios I-II-III, que clínicamente pueden ser tratados pasan a tratamiento quirúrgico.

Los carcinomas escamosos de esófago entran en el protocolo terapéutico (Fig. 4).

\section{PROTOCOLO CÁNCER DE ESÓFAGO}

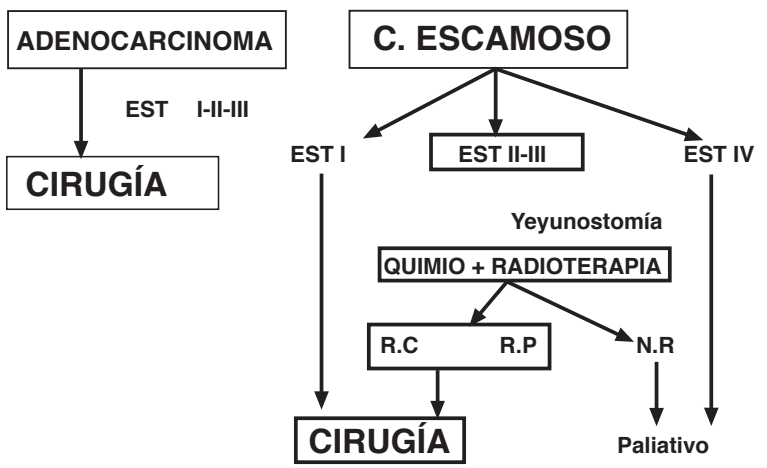

Fig. 4.

Una vez estudiado el paciente y el primer ingreso, bajo anestesia general se le coloca un reservorio venoso subcutáneo en el lado derecho para administrar posteriormente la quimioterapia. A través de una incisión para rectal superior izquierda, se localiza el ángulo de Treitz y en la primera asa yeyunal isoperistáltica se coloca una sonda de Foley (12 Fr) como yeyunostomía para alimentación, con tunelización a lo Witzel $(8 \mathrm{~cm})$, salida cutánea por orificio contralateral, pexia a la pared intrabdominal con tres puntos (anterior y posterior al orificio de la sonda y fijando la tunelización).

El paciente es dado de alta hospitalaria con nutrición enteral domiciliaria $2.000 \mathrm{cc} /$ día (dieta normocalórica, administrada con bomba $80 \mathrm{cc} / \mathrm{h}$ ) y en función del grado de disfagia se adaptará su dieta. Los objetivos por los cuales se coloca dicha sonda son: asegurar de forma continua el aporte calórico a dichos pacientes, para que no pierdan más peso durante el tratamiento, evitar reingresos por la toxicidad del tratamiento, garantizar que finalizarán el tratamiento y su respuesta terapéutica será mejor garantizando el aporte nutricional; en los pacientes que no exista respuesta el tratamiento será definitivo.
Se elige la yeyunostomía para preservar el estómago para una posible gastroplastia posterior; además, por esta vía el paciente no presenta reflujo ni vómitos y la toxicidad no afecta a la motilidad yeyunal. Se debe controlar la velocidad de perfusión para que el paciente se adapte y no presente diarreas.

Dicho procedimiento se estandarizó en el segundo año del protocolo a la vista de la toxicidad que afectaba a la calidad de vida del paciente y se obligaba a continuos reingresos.

El paciente es presentado y valorado por el Comité de tumores (Grupo Cáncer de Esófago H.U.G.TiP.).

En función de sus criterios de resecabilidad, el paciente se incluye en un grupo A, Quimiorradioterapia preoperatoria o grupo B, Quimiorradioterapia radical.

\section{Criterios de inclusión}

La confirmación histológica de carcinoma escamoso, estadiaje II o III, grados 0, 1 y 2 de la World Health Organization (WHO), mayores de 18 años, Karnofsky $>60 \%$, niveles de bilirrubina sérica menores de 1,5 mg/dl, creatinina sérica menor de $1.2 \mathrm{mg} / \mathrm{dl}$, recuento leucocitario mayor de $4.000 / \mathrm{ml}$ y más de $100.000 / \mathrm{ml}$ plaquetas. Se excluyeron los pacientes con fístula traqueobronquial, con metástasis a distancia así como aquellos pacientes con nódulos linfáticos cervicales o supraclaviculares y los pacientes con insuficiencias orgánicas descompensadas.

\section{Quimiorradioterapia preoperatoria}

Se administró radioterapia hiperfraccionada usando 6-10 MV fotones desde un acelerador lineal. Los campos de tratamiento fueron de un mínimo de $5 \mathrm{~cm}$ en el margen longitudinal y de $2 \mathrm{~cm}$ en el margen lateral del volumen de masa tumoral determinada por el esofagograma y el TC. La zona supraclavicular se incluyó en aquellos tumores localizados en los $2 / 3$ superiores del esófago torácico, mientras que la irradiación de los nódulos linfáticos celíacos se realizó a criterio de radioterapeuta. Cada paciente recibió una dosis total de $45 \mathrm{~Gy}$ en 30 fracciones de 1,5Gy, 2 fracciones por día con un intervalo de 6 horas cinco días por semana durante 3 semanas.

La pauta de quimioterapia consiste en cisplatino 30 $\mathrm{mg} / \mathrm{m}^{2}$ endovenoso durante 30 minutos administrado de forma semanal y $5-\mathrm{Fu} 300 \mathrm{mg} / \mathrm{m}^{2} /$ día en infusión continua endovenosa durante el período de la radioterapia.

Finalizado el tratamiento el Comité valora su respuesta.

\section{Criterios de respuesta y toxicidad}

Todo el estudio, a excepción de la ecografía transesofági$\mathrm{ca}$, se repitió después del tratamiento. Definimos respuesta completa (RC) cuando el esofagograma era normal, no había evidencias de tumor en la endoscopia y el TC no evidencia patología; respuesta parcial (RP) cuando por el esofagograma y por endoscopia se evidencia más del $50 \%$ de regresión tumoral y sin cambios (NC), cuando la evidencia de regresión tumoral era menor del $50 \%$. Después de la intervención los pacientes fueron estudiados según los criterios de la UICC. La toxicidad inducida por el tratamiento se evaluó según los criterios de la WHO.

Los pacientes con respuesta completa o respuesta parcial al tratamiento, a las cuatro semanas pasan a Cirugía. 


\section{J. Fernández-Llamazares y cols.}

\section{Cirugía}

El mismo grupo de anestesia, equipo quirúrgico y técnica realizada.

Con el paciente en decúbito lateral izquierdo, se practica una toracotomía derecha a través del $5^{\circ}$ espacio intercostal realizando la esofagolisis torácica y la linfadenectomía (mínimo 15 adenopatías) dejando dos drenajes torácicos. Se coloca el paciente en decúbito supino; a través de una laparotomía media suprainfraunbilical, se realiza la gastrolisis y linfadenectomia abdominal. Mediante una cervicotomía izquierda localizamos el esófago cervical seccionándolo, extrayendo el esófago torácico con su tumoración residual por vía abdominal. El estómago es tubulizado (según técnica de Akiyama), se amplía el hiato esofágico (según técnica de Pinotti), realizamos pilorotomía extramucosa y piloroplastia (3 puntos). La plastia la ascendemos por tracción de sonda nasogástrica, a través del mediastino hasta el cuello. A nivel cervical realizamos la anastomosis esófago gástrica termino terminal con material irreabsorvible. Se deja drenaje de Penrose cervical, sonda nasogástrica, cerrando la cervicotomía. El paciente ya es portador de una yeyunostomía que se conserva; si no, se coloca. El paciente pasa a la Unidad de críticos con dos objetivos básicos, la extubación precoz y bomba de PCA para el dolor.

\section{Análisis estadístico}

La supervivencia se ha calculado mediante el método de Kaplan-Meyer y la diferencia entre la supervivencia de ambos grupos mediante el test long rank. Para evaluar las características de los pacientes y la respuesta clínica se ha utilizado la $x^{2}$.

\section{Pacientes}

Desde enero de 1997 hasta enero de 2003 han sido tratados en nuestro centro 143 pacientes afectos de neoplasia de esófago.

El 30\% (46 casos) adenocarcinomas, de los cuales después del estudio de extensión se ha resecado el 66\% (30) con una mortalidad del 6,5\%. El 70\% (97 casos) de nuestros pacientes tenía un carcinoma de esófago epidermoide. Después del estudio de extensión, el $29 \%$ (28 casos) fue diagnosticado de EST-IV; por edad avanzada y mal estado general fue excluido el $7 \%$ (7 casos). Entraron en el protocolo para quimioterapia+radioterapia preoperatorio 62 casos $(63 \%$ de los tumores epidermoides), el (43\% de los tumores esofágicos).

Estos 62 pacientes con carcinoma escamoso de esófago eran 56 hombres (89\%) y 6 mujeres (11\%), con una edad media de 61 años y un rango $42-76$ años. La localización de la lesión fue: $1 / 3$ superior en 6 casos (10\%), 1/3 medio 48 casos $(75 \%)$ y $1 / 3$ inferior 8 casos (15\%). Su estadio preoperatorio: estadio II-A 5 casos (7\%), estadio II-B 1 caso (2\%), estadio III 56 casos (91\%).

\section{Resultados}

\section{Toxicidad}

Valoramos la toxicidad inducida por el tratamiento según la clasificación de Who.
La leucopenia en Grado 1ำ en el $11 \%$ de los casos, en el

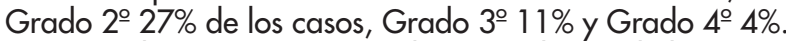

La trombocitopenia en Grado $1 \%$ en el $55 \%$ de los casos y en Grado 2 $5 \%$.

La anemia en Grado 1을 $22 \%$ de los casos y en Grado $2^{\circ}$ $11 \%$.

Las náuseas y los vómitos fueron bien controlados.

La esofagitis estuvo presente en Grado $2^{\circ} 16 \%$ de los casos, en Grado 3우 $38 \%$ de los casos y en Grado $4^{\circ} 27 \%$ de los pacientes.

No se registraron signos de nefrotoxicidad ni diarreas.

Desde que se introdujo en el protocolo la colocación sistemática de la yeyunostomía de alimentación, descendieron drásticamente los reingresos en esta fase del tratamiento, manteniendo el $90 \%$ de nuestros pacientes un peso estable.

\section{Respuesta}

El tratamiento preoperatorio completo fue realizado por el $79 \%$ de los pacientes (48 casos).

El Comité de tumores (Grupo Cáncer de Esófago H.U.G.TiP.), siguiendo el protocolo establecido valoró la respuesta al tratamiento, así como el estado general del paciente y sus riesgos quirúrgicos. $(56 \%)$.

La indicación de esofaguectomía fue para 35 casos

\section{Cirugía}

De los 35 pacientes que fueron intervenidos, esofaguectomía en 34 casos con reconstrucción gástrica según técnica descrita. Mortalidad 8\% (3 casos), todos de causa respiratoria.

\section{Hallazgos histológicos}

En el análisis de la respuesta al tratamiento valorando el material de resección podemos ver: PCR (respuesta completa patológica) 14 casos (40\%); Respuesta parcial: presencia de tumor microscópico 9 casos (25\%), p $\mathrm{pT}_{0} \mathrm{~N}_{1}$ en 3 casos (9\%).

En conjunto, la respuesta parcial es del $34 \%$ (12 casos).

No respuesta: $\mathrm{pT}_{2} \mathrm{~N}_{2}$ en 1 caso, $\mathrm{pT}_{3} \mathrm{~N}_{1}$ en 4 casos,

$\mathrm{pT}_{4} \mathrm{~N}_{1} \mathrm{M}_{1}$ en 1 caso.

En conjunto, sin respuesta $17 \%$ (6 casos).

\section{Estado actual de los pacientes.}

Con un seguimiento medio de 46 meses (12-72) el estado actual de los pacientes es el siguiente: de los 35 pacientes operados, en la actualidad viven 15 asintomáticos, habiendo fallecido en el transcurso de su enfermedad los 20 restantes. Hemos de destacar que los pacientes vivos libres de enfermedad son aquellos que presentaron una respuesta completa patológica $^{21}$.

De los 13 pacientes no operados que sólo han recibido tratamiento con quimioradioterapia, viven 5 asintomáticos y 8 han fallecido a consecuencia de su enfermedad.

Si comparamos la supervivencia de nuestros pacientes en la actualidad con el protocolo preoperatorio de quimio y radioterapia+cirugía, frente a nuestros resultados de supervivencia previos con sólo cirugía, podemos observar (Fig. 5) cómo en la actualidad ha mejorado de forma significativa $(p<0.01)$ dicha supervivencia, tanto a los tres años, en que 


\section{Supervivencia Ca. Escamoso Resecado a los 3 años}

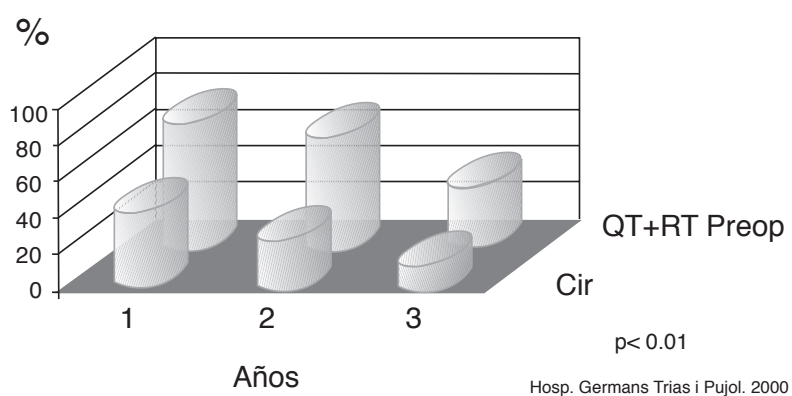

Fig. 5 .

\section{Supervivencia a los 5 años}

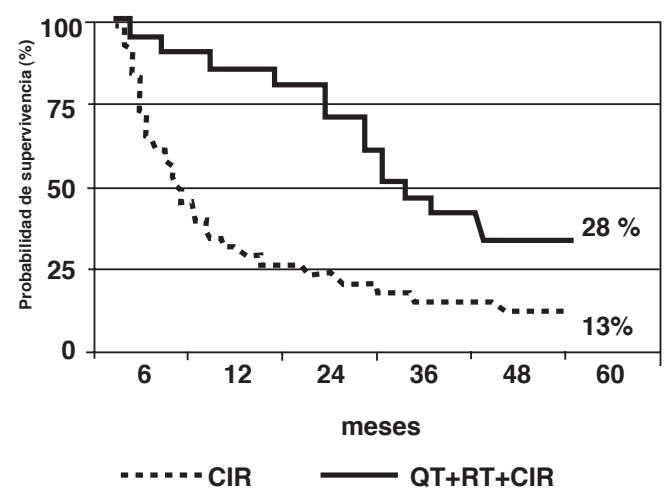

Hosp. Germans Trias i Pujol. 2003

Fig. 6 .

prácticamente ha pasado a ser el doble, como a los cinco años, en que la tasa de supervivencia de cirugía del 13 pasa al $28 \%$ con quimioterapia, radioterapia y cirugía.

Este es el principal de los objetivos que planteaba nuestro estudio.

Evidentemente, el problema de la supervivencia de dichos tumores no está resuelta, pero creemos que el tratamiento adyuvante será su solución en el momento que se encuentre una asociación terapéutica que beneficie a un mayor número de pacientes.

\section{Bibliografía}

1. Jemal A, Murria T, Samuels A, Ghafoor A, Ward E, Thun MJ. Cancer statistics, 2003. CA Cancer J Clin 2003; 53:5-26.

2. Altorki Nasser and Skinner David. Should en bloc esophagectomy be the standard of care for esophageal carcinoma? Ann Surg 2001; 234(5):581-9.

3. Hulscher JB, van Sandick JW, de Boer AGEM et als. Extended Transthoracic resection compared with limited transhiatal resection for Adenocarcinoma of the esophagus. N Engl J Med 2002; 347:1662-9.
4. Reed CE, Eloubeidi MA. Nuevas técnicas para la asignación de la etapa de cancer de esófago. Sur Clin N.A. Ed. Mc Graw Hill 2002; 4:673-87.

5. Mariette Ch, Finzi L, Fabre S, Balon JM, Van Seuningen I, Triboulet JP. Factors predictive of complete resection of operable esophageal cancer: A prospective study. Ann Torac Surg 2003; 75:1720-6.

6. Enzinger PC, Mayer RJ. Esophageal cancer. N Engl J Med 2003; 349(2):2241-52.

7. Litle VR, Buenaventura P O,Luketich JD. Resección muy poco invasiva del cancer de esófago. Sur Clin NA. Ed. Mc Graw Hill 2002; 4:687-705.

8. Rafaely J, Krasna MJ. Terapéutica de modalidades múltiples para el cancer de esófago. Sur Clin NA. Ed. Mc Graw Hill 2002; 4:705-23.

9. Burmeister BH, Smithers BM, Fitzgerald L, et al. A randomized phase III trial of preoperative chemoradiation followed by surgery (CR-S) versus surgery alone (S) for localized resectable cancer of the esophagus. Prog Proc Am Soc Clin Oncol 2002; 21:130 a.

10. Urba $S G$, Orringer $M B$, Turrisi $A$, lannetoni $M$, Forastiere $A$, Strawderman M. Randomized trial of preoperative chemoradiation versus surgery alone in patients with locoregional esophageal carcinoma. J Clin Oncol 2001; 19:305-13.

11. Nygaard K, Hagen S, hansen HS, et al. Pre-operative radiotherapy prolongs survival in operable esophageal carcinoma: $A$ randomized, multicenter study of pre-operative radiotherapy and chemotherapy: the second Scandinavian trial in esophageal carcinoma. World J Surg 1992; 16:1104-10.

12. Bosset JF, Gignoux M, Triboulet JP, et al. Chemoradiotherapy followed by surgery compared with surgery alone in squamouscell cancer of the esophagus. N Engl J Med 1997; 337:161-7.

13. Le Prise E, Etienne PL, Meunier B, et al. A randomized study of chemotherapy, radiation therapy, and surgery versus surgery for localized squamous cell carcinoma of the esophagus. Cancer 1994; 73:1179-84.

14. Apinop C, Puttisak P, Percha N. A prospective study of combined therapy in esophageal cancer. Hepatogastroenterology 1994; 41:391-3.

15. Walsh T, Noonan N, Hollywood D, Kelly A, Keeling N, Hennessy TPJ. A comparison of multimodal therapy and surgery for esophageal adenocarcinoma. N Engl J Med 1996; 335:462-7.

16. Law $S$, Kwong $D$, Tung $H$, et al. Preoperative chemoradiation for squamous cell esophageal cancer: a prospective randomized trial. Can J Gastroenterol 1998; 12:suppl B:56b.

17. Birkmeyer JD, Stukel TA, Siewers AE, Goodney PP, Wenneberg $D E$, Lucas FL. Surgeon volume and operative mortality in the United States. N Engl J Med 2003; 349(22): 2117-61.

18. Font A, Fernández-LLamazares J, Arellano A, Casas D, Boix J, Mesalles E, Abad A, Rosell R. Multinodal treatment for squamous cell carcinoma of the esophagus: Results with two different strategies. Rev Oncología 2001; 3(3):56-78.

19. Kelsen DP, Ginsberg R, Pajak TF, et al. Chemotherapy followed by surgery compared with surgery alone for localized esophageal cancer. N Engl J Med 1998; 339:1979-84.

20. Siewert JR, Stein HJ,Feith M, Bruecher BL, Barteis H, Fink U. Histologic tumor type is an independent prognostic parameter in esophageal cancer: Lessons from more than 1,000 consecutive resections a single center in the Western world" Ann Surg 2001; 234(3):360-7.

21. Siewert JR, OH K. Multimodality therapy concepts in esophageal carcinoma. Kongressbd Dłsch Ges Chir Kongr 2001; 118: 39-43. 